$\mathrm{J}$ o u r n a l of

Mathematics

and Applications

JMA No 44, pp 119-122 (2021)

\title{
A Sandwich Type Hahn-Banach Theorem for Convex and Concave Functionals
}

\author{
Jingshi $X u$
}

ABstract: We give a sandwich type Hahn-Banach theorem for convex and concave functionals.

AMS Subject Classification: 46A22.

Keywords and Phrases: The Hahn-Banach theorem; Convex functional; Concave functional.

The Hahn-Banach theorem is a fundamental theorem in linear functional analysis. Its sandwich form is the following, see Theorem 3.9 in [5].

Theorem 1 (Sandwich Theorem). Let $g: X \rightarrow \mathbb{R} \cup\{+\infty\}$, and $h: X \rightarrow \mathbb{R}$ be sublinear functions on a linear space $X$. If $-g \leqslant h$, there exists a linear form $l$ on $X$ such that $-g \leqslant l \leqslant h$.

The following Hahn-Banach extension theorem was given in [1] and [3].

Theorem 2. Suppose $X$ is a real linear space, $p$ is a convex functional on $X, M$ is a subspace of $X$. If $g$ is a real linear functional on $M$ such that $g(x) \leqslant p(x), x \in M$, then there exists a linear functional $f$ on $X$ such that $f(x) \leqslant p(x), \forall x \in X$ and $f(x)=g(x), \forall x \in M$.

In the following we shall use 0 to denote both zero and zero vector. From Theorem 2 , we have the following results.

Corollary 1. Let $X$ be a real linear space and $\varphi$ be a convex functional on $X$ such that $\varphi(0) \geqslant 0$, then there exists a linear functional $L$ on $X$ such that $L(x) \leqslant \varphi(x)$ for every $x \in X$.

Proof. Let $E=\{0\}$ and $f_{0}(0)=0$, The $f_{0}$ is a linear functional on $E$ such that $f_{0}(x) \leqslant \varphi(x)$ for every $x \in E$. Then by Theorem 2 , there exists a linear functional $f$ on $X$ such that $f(x) \leqslant \varphi(x)$ for every $x \in X$.

COPYRIGHT (C) by Publishing House of Rzeszów University of Technology P.O. Box 85, 35-959 Rzeszów, Poland 
Corollary 2. Suppose that $f_{0}$ be a linear functional on subspace $M$ of $X$, such that $\psi(x) \leqslant f_{0}(x)$ for every $x \in M$, where $\psi$ is a concave function on $X$. Then there exists a linear functional $L$ on $X$ such that $L(x)=f_{0}(x)$ for every $x \in M$ and $\psi(x) \leqslant L(x)$ for every $x \in X$.

Now, our main result is the following sandwich type theorem for convex and concave functionals.

Theorem 3. Let $M$ be a subspace in $X$. Suppose $\varphi$ and $-\psi$ are convex functionals on $X$ such that $\varphi(0)=\psi(0)=0$ and $T(x):=\inf _{y \in X}\{\varphi(x+y)-\psi(y)\}$ is finite for every $x \in X$. If $f_{0}$ is a linear functional on $M$, then there exists an extension linear functional $L$ on $X$ of $f_{0}$ such that $\psi(x) \leqslant L(x) \leqslant \varphi(x)$ for every $x \in X$ if and only if $f_{0}(x) \leqslant T(x)$ for every $x \in M$.

To give the proof of Theorem 3, we need the following lemmas.

Lemma 1. Suppose $\varphi$ and $-\psi$ are convex functionals on $X$ such that $\varphi(0)=\psi(0)=0$ and $T(x):=\inf _{y \in X}\{\varphi(x+y)-\psi(y)\}$ is finite for every $x \in X$. Let $f_{0}$ be a linear functional on a subspace $M$ of $X$ such that

$$
f_{0}(x) \leqslant T(x) \text { for every } x \in M .
$$

Then the following conditions are satisfied.

(i) For every $x \in X, \psi(x) \leqslant \varphi(x)$;

(ii) For every $x \in M, \psi(x) \leqslant f_{0}(x) \leqslant \varphi(x)$.

Proof. From (1), for every $y \in X$ and $x \in M, f_{0}(x) \leqslant \varphi(x+y)-\psi(y)$. Then, let $x=0$, we have $\psi(y) \leqslant \varphi(y)$ for every $y \in X$. By letting $y=0$, we see that $f_{0}(x) \leqslant \varphi(x)-\psi(0) \leqslant \varphi(x)$ for every $x \in M$. By letting $y=-x$, we obtain that $f_{0}(-y) \leqslant-\psi(y)$ or $\psi(x) \leqslant f_{0}(x)$ for every $x \in M$. Thus, $\psi(x) \leqslant f_{0}(x) \leqslant \varphi(x)$ for every $x \in M$.

Lemma 2. Suppose $\varphi$ and $-\psi$ are convex functionals on $X$ such that $\varphi(0)=\psi(0)=0$ and $T(x):=\inf _{y \in X}\{\varphi(x+y)-\psi(y)\}$ is finite for every $x \in X$. Let $\psi(x) \leqslant \varphi(x)$ for every $x \in X$. Then $\psi(x) \leqslant T(x) \leqslant \varphi(x)$ for every $x \in X$, and $T$ is a convex functional. Moreover, if $L$ is a linear functional on $X$ such that $\psi(x) \leqslant L(x) \leqslant \varphi(x)$ for every $x \in X$, then $L(x) \leqslant T(x)$ for every $x \in X$.

Proof. First, we prove that $T$ is convex. Fix $u, v \in X$. For $\alpha, \beta \geq 0$ such that $\alpha+\beta=1$, for every $\epsilon>0$, there exist $y, z \in X$ such that $\varphi(u+y)-\psi(y)<T(u)+\epsilon$, $\varphi(v+z)-\psi(z)<T(v)+\epsilon$, then

$$
\begin{aligned}
\varphi(\alpha u+\beta v+\alpha y+\beta z)-\psi(\alpha y+\beta z)\} & \leqslant \alpha \varphi(u+y)+\beta(v+z)-\alpha \psi(y)-\beta \psi(z) \\
& \leqslant \alpha(\varphi(u+y)-\psi(y))+\beta(\varphi(v+z)-\psi(z)) \\
& <\alpha T(u)+\beta T(v)+\epsilon .
\end{aligned}
$$

Thus $T(\alpha u+\beta v)<\alpha T(u)+\beta T(v)+\epsilon$. Since $\epsilon$ is arbitrary, we obtain that $T(\alpha u+\beta v) \leqslant$ $\alpha T(u)+\beta T(v)$. Therefore $T$ is convex. 
Since $T(x) \leqslant \varphi(x+y)-\psi(y)$, it follows that $T(x) \leqslant \varphi(x)-\psi(0)$. So $T(x) \leqslant \varphi(x)$ for every $x \in X$. Again, $T(-y) \leqslant \varphi(0)-\psi(y)$, So $T(-y) \leqslant-\psi(y)$. Since $T(0)=0$, and by the convexity of $T, 0 \leqslant T(0) \leqslant 1 / 2 T(y)+1 / 2 T(-y)$, so that $-T(y) \leqslant T(-y)$. Hence, $-T(y) \leqslant T(-y) \leqslant-\psi(y)$. Thus $\psi(y) \leqslant T(y)$ for every $y \in X$. Consequently, $\psi(x) \leqslant T(x) \leqslant \varphi(x)$ for every $x \in X$.

Finally, suppose that $\psi(x) \leqslant L(x) \leqslant \varphi(x)$ for every $x \in X$. Now $\psi(u) \leqslant L(u)$, it follows that $L(u) \leqslant-\psi(-u)$. Hence, by the linearity of $L$ we obtain that $L(u+$ $v) \leqslant \varphi(v)-\psi(-u)$ for every $u, v \in X$. Letting $v=x+y$ and $u=-y$, we obtain $L(x) \leqslant \varphi(x+y)-\psi(y)$. Taking the infimum over all $y \in X$, we obtain that $L(x) \leqslant T(x)$ for every $x \in X$.

Proof of Theorem 3. If a linear functional $L$ on $X$ is an extension of $f_{0}$ such that $\psi(x) \leqslant L(x) \leqslant \varphi(x)$ for every $x \in X$. By Lemma $2, L(x) \leqslant T(x)$ for every $x \in X$. Since $f_{0}(x)=L(x)$ for each $x \in M$, so $f_{0}(x) \leqslant T(x)$ for every $x \in M$.

Conversely, if $f_{0}(x) \leqslant T(x)$ for every $x \in M$, by Lemma $1, \psi(x) \leqslant \varphi(x)$ for all $x \in X$ and $\psi(x) \leqslant f_{0}(x) \leqslant \varphi(x)$ for all $x \in M$. According to Lemma 2 , we see that $T$ is a convex function. Now, by Theorem 2 there is an extension linear functional $L$ on $X$ such that $f_{0}(x)=L(x)$ for each $x \in M$ and $L(x) \leqslant T(x)$ for each $x \in X$. By Lemma $1, \psi(x) \leqslant L(x) \leqslant \varphi(x)$ for all $x \in X$.

By Theorem 3, we have an generalization of Theorem 1 as follows.

Theorem 4. Suppose $\varphi$ and $-\psi$ are convex functionals on $X$ such that $\varphi(0)=\psi(0)=$ 0 and $T(x):=\inf _{y \in X}\{\varphi(x+y)-\psi(y)\}$ is finite for every $x \in X$. If $\psi(x) \leqslant \varphi(x)$ for every $x \in X$, then there exists a linear functional $L$ on $X$ such that $\psi(x) \leqslant L(x) \leqslant$ $\varphi(x)$ for every $x \in X$.

Proof. Let $E=\{0\}$ and $f_{0}(0)=0$, The $f_{0}$ is a linear functional on $E$ such that $\psi(x) \leqslant f_{0}(x) \leqslant \varphi(x)$ for every $x \in E$. Then by Theorem 3 , there exists a linear functional $f$ on $X$ such that $\psi(x) \leqslant f(x) \leqslant \varphi(x)$ for every $x \in X$.

In Theorems 3 and 4 , the condition $\varphi(0)=\psi(0)=0$ is necessary. For example, in $\mathbb{R}$, let $\varphi(x)=(x+4)^{2}-4, \psi(x)=-e^{x}-4$, then there exists no constant $k$ such that $\varphi(x) \geqslant k x \geqslant \psi(x)$ for all $x \in \mathbb{R}$.

Remark 1. Theorem 4 partly generalises the sandwich version Hahn-Banach Theorem in [2]. Páles gave a different type Sandwich theorems in [4].

\section{Acknowledgments}

The author would like to thank the referees for their careful reading and suggestions, in particular, the reference [3] was pointed by them.

\section{References}

[1] L. Bittner, A remark concerning Hahn-Banach's extension theorem and the quasilinearization of convex functionals, Math. Nachr. 51 (1971) 357-372. 
[2] M. Lassonde, Hahn-Banach theorems for convex functions, In: Ricceri B., Simons S. (eds) Minimax Theory and Applications, Nonconvex Optimization and Its Applications, vol. 26, 135-145, Springer, 1998.

[3] S. Mazur, Über konvexe Mengen in linearen normierten Räumen, Studia Math. 4 (1933) 70-84.

[4] Z. Páles, Separation theorems for convex sets and convex functions with invariance properties, In: Hadjisavvas N., Martinez-Legaz J.E., Penot JP. (eds) Generalized Convexity and Generalized Monotonicity, Lecture Notes in Economics and Mathematical Systems 502, 279-293, Springer, Berlin, 2001.

[5] J.P. Penot, Analysis From Concepts to Applications, Switzerland: Springer International Publishing, 2016.

DOI: $10.7862 / \mathrm{rf} .2021 .9$

\section{Jingshi Xu}

email: jingshixu@126.com

ORCID: 0000-0002-5345-8950

School of Mathematics and Computing Science

Guilin University of Electronic Technology

Guilin 541004

CHINA

Received 24.04.2020

Accepted 26.08.2021 